INPLASY

PROTOCOL

To cite: He et al. Comparative efficacy of AcupunctureRelated Techniques for urinary retention after spinal cord injury: a Bayesian network meta-analysis. Inplasy protocol 2021110005. doi:

10.37766/inplasy2021.11.0005

Received: 02 November 2021

Published: 02 November 2021

Corresponding author:

Ruijie Ma

maria7878@sina.com

Author Affiliation:

Zhejiang Chinese Medical

University.

Support: Ruijie Ma.

Review Stage at time of this submission: Completed but not published.

Conflicts of interest: None declared.

\section{Comparative efficacy of Acupuncture- Related Techniques for urinary retention after spinal cord injury: a Bayesian network meta-analysis}

He, K¹; Li, X²; Qiu, B3; Jin, L4; Ma, R5.

Review question / Objective: Urinary retention refers to impaired voiding despite a full bladder, which leads to an elevated postvoid residual urine volume (PVR), there is a strong demand to develop novel and effective therapies. Acupuncture as an integral part of traditional medicines throughout the world to management urinary retention has recently drawn widespread attention and increasingly applied in clinical and scientific research. Although there are many acupuncture-related interventions for urinary retention after $\mathrm{SCl}$, there is still a lack of comparison on the efficacy of different acupuncture interventions and the optimal choice of acupuncture is still uncertain.Therefore, this study aimed to use NMA to explore the efficacy of different acupuncture therapy against urinary retention after SCI. Finally, the study may help to provide guidelines for the acupuncture therapy of urinary retention after $\mathrm{SCl}$ and a perspective for the future.

INPLASY registration number: This protocol was registered with the International Platform of Registered Systematic Review and Meta-Analysis Protocols (INPLASY) on 02 November 2021 and was last updated on 02 November 2021 (registration number INPLASY2021110005).

\section{INTRODUCTION}

Review question / Objective: Urinary retention refers to impaired voiding despite a full bladder, which leads to an elevated postvoid residual urine volume (PVR), there is a strong demand to develop novel and effective therapies. Acupuncture as an integral part of traditional medicines throughout the world to management urinary retention has recently drawn widespread attention and increasingly 
applied in clinical and scientific research. Although there are many acupuncturerelated interventions for urinary retention after $\mathrm{SCl}$, there is still a lack of comparison on the efficacy of different acupuncture interventions and the optimal choice of acupuncture is still uncertain.Therefore, this study aimed to use NMA to explore the efficacy of different acupuncture therapy against urinary retention after SCI. Finally, the study may help to provide guidelines for the acupuncture therapy of urinary retention after $\mathrm{SCl}$ and a perspective for the future.

Condition being studied: Acupuncture as an integral part of traditional medicines throughout the world to management urinary retention has recently drawn widespread attention and increasingly applied in clinical and scientific research. Moreover, acupuncture has advantages such as safety, convenience, very few side effects, and economical. In China, Acupuncture, with many categories such as manual acupuncture, electroacupuncture, moxibustion therapy, auricular acupuncture and acupoint patching, are widely used to the treatment of urinary retention after $\mathrm{SCl}$. Although there are many acupuncture-related interventions for urinary retention after $\mathrm{SCl}$, there is still a lack of comparison on the efficacy of different acupuncture interventions and the optimal choice of acupuncture is still uncertain.Urinary retention refers to impaired voiding despite a full bladder, which leads to an elevated postvoid residual urine volume (PVR), there is a strong demand to develop novel and effective therapies. Acupuncture as an integral part of traditional medicines throughout the world to management urinary retention has recently drawn widespread attention and increasingly applied in clinical and scientific research. Although there are many acupuncturerelated interventions for urinary retention after $\mathrm{SCl}$, there is still a lack of comparison on the efficacy of different acupuncture interventions and the optimal choice of acupuncture is still uncertain.

\section{METHODS}

Search strategy: Terms:"spinal cord injuries", "spinal cord injury", "urinary retention", "neurogenic bladder", "acupuncture", "acupuncture therapy", "electroacupuncture", "warming needle moxibustion", "moxibustion", "acupoint injection", "acupoint patching", "auricular acupuncture", "ear acupuncture". Electronic databases: Pubmed, Cochrane library, Web of science, China national knowledge infrastructure (CNKI), Chinese Biomedical Literature database (CBM), Wan-fang database and the Chinese Scientific Journals Full-text Database (VIP).

Participant or population: Adult participants (>18years of age) diagnosed as $\mathrm{SCl}$-induced urinary retention.

Intervention: Acupuncture alone or in combination with conventional therapy.

Comparator: conventional therapy, conventional therapy and sham acupuncture, conventional therapy and other acupuncture.

Study designs to be included: Systematic review with network meta-analyses involving multidimensional outcomes

Eligibility criteria: This study will included all published randomized controlled trials (RCTs). Any other studies, such as review, animal studies, case reports, case series, letters, comments, non-clinical trials, nonRCTs, and quasi-RCTs studies were all excluded.

Information sources: Pubmed, Cochrane library, Web of science, CNKI, CBM, Wanfang database and VIP, to identify all the RCTs on acupuncture in treating SClinduced urinary retention. Any unclear information will contact with corresponding author and trial registers.

Main outcome(s): Clinical effectiveness rate.

Additional outcome(s): Residual urine volume. 
Quality assessment / Risk of bias analysis: Cochrane risk of bias assessment tool.

Strategy of data synthesis: The combined results were presented as odds ratios (ORs) with $95 \%$ confidence intervals $(95 \%$ CIs) for dichotomous outcomes.The combined results were presented as mean differences (MDs) with $95 \%$ Cis for continuous outcomes.

Subgroup analysis: Because the study was a network meta-analysis, subgroup analysis was not obligatory.

Sensitivity analysis: Because the study was a network meta-analysis, subgroup analysis was not obligatory.

Country(ies) involved: China.

Keywords: Acupuncture, Spinal cord injury, Urinary retention, Network meta-analysis, Clinically effective.

Contributions of each author:

Author 1 - Kelin He.

Email: 352128492@qq.com

Author 2 - Xinyun Li.

Email: Ixyjasmine2010@163.com

Author 3 - Bei Qiu.

Email: 1109476936@qq.com

Author 4 - Linzhen Jin.

Email: 978249839@qq.com

Author 5 - Ruijie Ma.

Email: maria7878@sina.com 